\title{
Planejamento de Recursos Humanos de Saúde e Ambiente - aspectos históricos, teóricos e reflexões para políticas públicas em Vigilância em Saúde Ambiental no Brasil.
}

Health and Environment - historical, theoretical and reflections for public policies in Environmental Health

Surveillance in Brazil.

Salud y Ambiente - histórico, teórico y reflexiones para las políticas públicas de Vigilancia de la Salud Ambiental en Brasil.

\author{
Juliana Wotzasek Rulli VILLARDI ${ }^{1}$ \\ Jorge Mesquista Huet MACHADO ${ }^{1}$ \\ Guilherme Franco NETTO ${ }^{1}$ \\ André Luiz Dutra FENNER ${ }^{1}$ \\ Daniela Buosi ROLFS ${ }^{2}$
}

RESUMO: As relações entre a saúde e o ambiente no âmbito das atribuições do Sistema Único de Saúde é um desafio no cuidado coletivo na perspectiva da identificação, prevenção, controle e das recomendações de processos envolvidos na exposição humana às situações ambientais que possam gerar impacto e consequências negativas para a saúde humana. As reflexões sobre os caminhos da vigilância em saúde ambiental (VSA), seu histórico, conceito, teoria e alternativas metodológicas, que geram informação para tomada de decisão e inclui possibilidades de interpretação e transformação da realidade são os objetivos abordados neste ensaio. A partir de uma pesquisa exploratória sobre a Vigilância em Saúde Ambiental no Brasil, suas práticas e teorias, foi realizada uma revisão sobre a vigilância em saúde ambiental no Brasil, e seu contexto teórico e histórico. O objeto da Vigilância em Saúde Ambiental é a relação sócio ecológica do ambiente, da saúde e da sustentabilidade, e há que se avançar e pensar na análise de situação de saúde ambiental como eixo da discussão do seu objeto produzindo informação qualificada para a intervenção solidária e democrática tendo a saúde como valor que traz a discussão da sustentabilidade, da equidade, com a incorporação de uma política social que interaja condicionando o desenvolvimento econômico e social.

Palavras-chave: Vigilância Sanitária Ambiental.Vigilância em Saúde Pública.Saúde Ambiental. Exposição Ambiental.

\footnotetext{
${ }^{1}$ Doutores em Ciências na área de Saúde Pública. Colaboradores do MS/SVS/DSAST - Brasília (DF), Brasil.Fiocruz. Email: julianarulli@gmail.com

${ }^{2}$ Mestre em Ciências Ambientais pela Universidade de Brasília (UnB); Coordenadora Geral de Vigilância em Saúde Ambiental da CGVAM/DSAST/SVS/MS - Brasília (DF), Brasil. 


\begin{abstract}
The relationship between health and the environment within the jurisdiction of the Unified Health System is challenging the collective care in connection with the identification, prevention, control and recommendations of processes involved in human exposure to environmental situations that generate impact and negative consequences to human health. The reflections about the environmental health surveillance (EHS), its historical, concept, theory and methodological alternatives that generate information for decision making and includes interpretation and transformation of reality Chances are the objectives addressed in this essay. From an exploratory research on Environmental Health Surveillance in Brazil, their practices and theories, a review of the surveillance was carried out in environmental health in Brazil, and its theoretical and historical context. The object of Environmental Health Surveillance is the relationship ecological social environment, health and sustainability, and we must move forward and think of the analysis of environmental health situation as axis discussing its object producing quality information for the joint intervention and democratic health as having the value it brings the discussion of sustainability, equity, with the incorporation of a social policy that interacts conditioning the economic and social development.
\end{abstract}

Keywords : Environmental Health Surveillance . Public Health Surveillance . Environmental Health .Environmental Exposure

RESUMEN: La relación entre la salud y el ambiente dentro de la jurisdicción del Sistema Único de Salud está desafiando la atención colectiva en relación con la identificación, prevención, control y recomendaciones de los procesos que intervienen en la exposición humana a las situaciones ambientales que generan impacto y consecuencias negativas para la salud humana. Las reflexiones sobre la vigilancia de la salud ambiental (VSA), su patrimonio histórico, el concepto, la teoría y alternativas metodológicas que generan información para la toma de decisiones e incluye la interpretación y transformación de la realidad más probable son los objetivos tratados en este ensayo. A partir de una investigación exploratoria sobre Vigilancia de Salud Ambiental en Brasil, sus prácticas y teorías, una revisión de la vigilancia efectuada en la salud ambiental en Brasil, y su contexto teórico e histórico. El objeto de Vigilancia de la Salud Ambiental es la relación ecológica entorno social, la salud y la sostenibilidad, y hay que seguir adelante y pensar en el análisis de situación de salud ambiental como eje discutir su objeto producir información de calidad para la intervención conjunta y salud democrática como tener el valor que aporta la discusión de la sostenibilidad, la equidad, con la incorporación de una política social que interactúa acondicionado el desarrollo económico y social. 
Palabras llave: Vigilancia Sanitaria Ambiental. Vigilancia de la Salud Pública . Salud Ambiental. Exposición ambiental .

\section{Introdução e alguns aspectos históricos para a Vigilância em Saúde Ambiental (VSA) no Brasil}

A ação humana em seu processo ecológico e no seu relacionamento com o meio ambiente, ao longo da história, principalmente na ação produtiva, altera o habitat e consequentemente o ecossistema, gerando exposição humana com possíveis efeitos deletérios à saúde. Como exemplo, tem-se as atividades agropecuárias que datam entre dez e doze mil anos; criaram situações de alteração ambiental, sendo considerada por alguns autores como passo decisivo para o desenvolvimento humano. Evidencia-se aqui grupos com objetivo diferente de produzir alimento, resultando em produção de novas tecnologias, acumulação de bens de capital, com o aculturamento de desenvolvimento como um aparente melhoramento de padrão de vida.

É principalmente por meio de Hipócrates (460-377 a.C.), e de sua obra, que se inicia uma abordagem racional para o conhecimento da exposição como fator fundamental para a medicina, entendendo o homem como parte da estrutura e do funcionamento do ecossistema. Os fatores ambientais tais como os climáticos, alimentares, dos lugares já eram destacados desde esta época na Grécia Antiga, em seus estudos, suas teorias e constatações com implicações para a saúde. Os grandes médicos gregos eram também filósofos naturais ${ }^{1}$. Durante a abordagem dos problemas de saúde, procuravam entender as relações entre o homem e a natureza. A explicação da saúde e da doença como resultantes de processos naturais e não sagrados eram as preocupações que estavam ali evidentes, e dessa forma a observação empírica da natureza forneceria os elementos para a organização do modo de conceber o adoecimento humano.

No final da Idade Média (séc. V `a XV) são criados códigos sanitários de forma a normatizar a localização de ambientes insalubres, tais como matadouros, o despejo de restos, o recolhimento do lixo, a pavimentação das ruas e a canalização de dejetos para poços cobertos ${ }^{1}$. A teoria miasmática ainda persistia como modelo explicativo, e a evidência que se tinha à época era a sugerida por Hipócrates, de uma alteração atmosférica, onde águas estagnadas e matéria orgânica em decomposição corrompiam o $\operatorname{ar}^{2}$.

O século XV foi marcado pela revalorização do saber técnico proveniente da agricultura, da mineração, da metalurgia e da navegação. As transformações na lógica produtiva, com o advento do capitalismo (que se inicia no século XVI) reorganiza-se a vida social, ainda que no campo da saúde. Porém no campo da saúde, nesta época, são desenvolvidos estudos de anatomia, físiologia e de individualização da descrição das doenças, fundada na observação clínica e epidemiológica.

Com fim do modelo feudal de sociedade e a expansão comercial, as cidades tornaram-se cada vez mais importantes do ponto de vista econômico e político. A produção torna-se elemento central na atividade econômica dos países. O conjunto de doutrinas políticas e econômicas do Estado Moderno tem o trabalho como elemento central de preocupação dos governos, influenciando diretamente a 
atuação da saúde pública. Foucault ${ }^{3}$ considera que é possível distinguir três etapas na formação da medicina social: (i) a medicina de Estado, surgida na Alemanha do século XVIII com a organização

de um sistema de observação da morbidade, com a normalização do saber e práticas médicas. Os médicos são subordinados à uma administração central; (ii) a medicina urbana, com seus métodos de vigilância e hospitalização, ainda que na prática não fosse mais do que um aperfeiçoamento do esquema político-médico da quarentena. Tinha como preocupação central a análise das regiões de amontoamento que significassem ameaça à saúde humana, como os cemitérios e os matadouros, propondo sua 're-localização' e (iii) o controle da circulação do ar e da água.

Ao longo dos séculos, até o século XIX, as ações transformadoras da natureza tiveram consequências localizadas, ou com pouca extensão. Num contexto de crescente urbanização dos países europeus e de consolidação do sistema de fábricas, a concepção de causação social passa a se fortalecer como interpretação das relações entre as condições de trabalho das populações e da classe trabalhadora. A criação de uma teoria social da medicina surge ao lado das condições objetivas da existência. O ambiente é a origem de todas as causas de doença quando deixa, momentaneamente, de ser natural para revestir-se do social. É nas condições de vida e trabalho do homem que as causa das doenças deverão ser buscadas ${ }^{4}$.

Por outro lado, em 1876, Robert $\mathrm{Koch}^{3}$ comprovou a existência de micoorganismos como a causa de uma determinada doença. Após a sua descoberta e publicação de seu artigo, muitos foram os avanços da medicina na lógica da intervenção ao agente patogênico. A microbiologia francesa de Pasteur e seus colaboradores concentraram esforços na descoberta dos mecanismos da infecção e nas formas de prevenção e tratamento das doenças contagiosas e ao final do século XIX boa parte das questões relacionadas às doenças infecciosas haviam sido respondidas especialmente tratando-se das às doenças contagiosas; mas também ocorre a consolidação da grande indústria e a universalização do sistema capitalista ampliando as formas de exposição humana e do seu adoecimento.

Nos primeiros anos do século XX, foram desvendados a participação de vetores ou hospedeiros intermediários na transmissão de doenças e o papel dos portadores sadios na manutenção da cadeia epidemiológica e ecológica. Vacinas e soros foram produzidos para um número expressivo de doenças. Os indicadores de mortalidade decrescem continuamente, e à medida que eram identificados os modos de transmissão e as formas de reprodução de vetores específicos, várias melhorias sanitárias e das condições de vida dos habitantes foram incentivadas. Porém o modelo social era excludente, centrado na exploração do trabalho, com impactos sobre o meio ambiente, consequentemente à saúde dos trabalhadores e da população geral ${ }^{5}$.

\footnotetext{
${ }^{3}$ Médico alemão, obedecendo aos postulados de Henle, demonstrou, durante três dias, diante de outros cientistas, a transmissão do antraz por um bacilo, usando camundongos como animais experimentais.
} 
Com um olhar centrado para o estudo do adoecimento no plano biológico firmou-se na lógica médica o pensamento hegemônico do orgânico. A racionalidade científica na medicina estava focada na explicação dos fenômenos com base no estudo de mudanças orgânicas, morfológicas e estruturais, nos corpos, órgãos e tecidos dos indivíduos. O modelo unicausal de compreensão da doença estava baseado na existência de apenas uma causa (o agente) para um agravo ou doença ${ }^{6}$ enquanto as transformações da vida e da dinâmica social eram outras e demandavam outros olhares.

Com a transição epidemiológica, caracterizada pela diminuição da importância das doenças infecto-parasitárias como causa de adoecimento e morte, e o aumento das doenças crônicodegenerativas, o modelo multicausal de doenças é favorecido e várias abordagens foram propostas para a compreensão do processo saúde-doença. O modelo ecológico, também conhecido como da história natural das doenças, proposto por Leavell e Clark (proposto em 1976) é fruto desta linha de pensamento e considera a interação, o relacionamento e o condicionamento de três elementos fundamentais da chamada 'tríade ecológica': o ambiente, o agente e o hospedeiro. A doença seria resultante de um desequilíbrio nas auto-regulações existentes no ecossistema. O exame dos diferentes fatores relacionados ao surgimento de uma doença e a utilização da estatística nos métodos de investigação e desenhos metodológicos permitiram significativos avanços na prevenção de doenças.

O modelo multicausal avançou no conhecimento dos fatores condicionantes da saúde e da doença. Segundo Palmeira ${ }^{7}$, a crítica que se faz a este modelo está em tratar todos os elementos da mesma forma, ou seja, naturalizar as relações entre o ambiente, o hospedeiro e o agente, sem considerar que o ser humano produz socialmente sua vida em um tempo histórico. Dessa forma podem ocorrer doenças diferentes com intensidades e manifestações também diferentes, a depender das características do dado território, sejam elas sociais, culturais, econômicas, geográficas, e até mesmo políticas.

Nesse sentido, ao olharmos a situação de saúde e ambiente atuais, há que levar em conta que o homem evoluiu ${ }^{4}$ através das eras históricas, sua forma de organização e sua relação com a natureza, ou o seu ambiente, sua forma de sociabilização e vivência em comunidade, sua arte e crenças, compreendendo uma complexa relação política, social, econômica, e cultural que deve ser levada em consideração para a promoção da sua saúde. As décadas de 60 e 70 do século XX foram marcadas pela substituição da noção de 'causalidade' do ponto de vista analítico, pela noção de 'determinação', com base na hierarquia das condições relacionadas à estrutura social. Está vinculada à compreensão dos 'modos e estilos de vida', derivados não só das escolhas pessoais, como de fatores culturais, práticas sociais e constituição do espaço.

Em consonância com o olhar das práticas de saúde, os problemas como energia, poluição, saneamento, tecnologia e crescimento populacional foram destacados na década de 70 por meio do relatório elaborado por uma equipe do Instituto de Tecnologia de Massachusetts (MIT),

\footnotetext{
${ }^{4}$ Evolução aqui no sentido do processo através no qual ocorrem mudanças ou transformações ao longo do tempo ISSN 1982-8829

Tempus actas de saúde colet. Brasília, 9(3), 105-120, set, 2015.
} 
contratada pelo Clube de Roma ${ }^{5}$, intitulado Os Limites do Crescimento. Na ocasião, utilizando modelos matemáticos, chegou-se à conclusão de que o Planeta Terra não suportaria o crescimento populacional devido à pressão gerada sobre os recursos naturais e energéticos e ao aumento da poluição, mesmo tendo em conta o avanço tecnológico. Ainda que nenhuma das previsões, tanto nos aspectos de esgotamento dos recursos naturais, como da evolução dos processos produtivos tenham se confirmado (há que se considerar que todo modelo matemático global existe imprecisões e simplificações), está colocada a observação de que o funcionamento dos ecossistemas está sendo modificado, e diferentes formas de olhar e de abordagens são necessárias, e certamente haverá os impactos à saúde. Na ocasião foram elencadas cinco grandes tendências de interesse global: (i) o ritmo acelerado da urbanização; (ii) o rápido crescimento demográfico; (iii) desnutrição; (iv) falta de recursos naturais não renováveis e (v) deterioração do ambiente ${ }^{8}$.

O modelo produtivo nesta época impulsiona o sistema econômico. As fábricas se multiplicam, substâncias químicas são incorporadas à produção ampliando diversificando os riscos à saúde dos trabalhadores e à população geral. Na agricultura, a revolução verde traz novas formas de produção de alimentos por meio de programa idealizado para aumentar a produção agrícola no mundo através da alteração genética de sementes, uso intensivo de insumos industriais, mecanização e redução do custo de manejo.

Dessa forma, em 1972 a Conferência das Nações Unidas sobre o Meio Ambiente Humano foi uma conferência no qual países se propunham a ajudar uns aos outros e fazer uma parcela de ajuda ao mundo. Foi a primeira atitude mundial em tentar organizar as relações do Homem e do Meio Ambiente. Realizada em Estocolmo, na Suécia, a sociedade científica já detectava graves problemas futuros por razão da poluição atmosférica provocada pelas indústrias. São realizadas as críticas ao crescimento econômico e às tecnologias associadas à ele, o mundo se direcionou para o volume da população absoluta global, a poluição atmosférica e a intensa exploração dos recursos nativos. Porém a discussão social, e as desigualdades na distribuição de renda ficaram à margem das temáticas propostas.

Em 1974, no Canadá, o relatório de nome A new perspective on the health of Canadians (Uma nova perspectiva da saúde de canadenses - tradução livre), denominado relatório Lalonde ${ }^{9}$ é considerado o primeiro relatório governamental moderno no mundo ocidental a reconhecer que a assistência médica sob um ponto de vista biomédico é restrita, sendo necessário um olhar para além do sistema tradicional de saúde (tratamento dos doentes). Lalonde ${ }^{6}$ propôs neste relatório que a saúde poderia ser classificada em quatro elementos gerais: biologia, ambiente, estilo de vida e organização da assistência sanitária. O relatório também enfatizou a responsabilidade de cada indivíduo em mudar seus comportamentos para melhorar sua saúde ${ }^{10}$, e também que intervenções em saúde pública deveriam dar ênfase em população de maior risco ${ }^{11}$.

\footnotetext{
${ }^{5}$ Grupo fundado em 1968 de pessoas ilustres que se reúnem para debater um vasto conjunto de assuntos relacionados a política, economia internacional e, sobretudo, ao meio ambiente e o desenvolvimento sustentável

${ }^{6}$ Marc Lalonde - Ministro de Bem-Estar e Saúde do Canadá à época. 
Outro marco importante foi a Declaração de Alma-Ata para os Cuidados Primários em Saúde, em 1978, incorporando as dimensões sociais, políticas, culturais e econômicas nesta relação. Os primeiros itens da declaração reafirmam a definição de saúde defendida pela Organização Mundial da Saúde (OMS), como o bem-estar físico, mental e social, e não simplesmente a ausência de doença, sendo a saúde considerada como direito fundamental e meta social de todos os governos.

A declaração foi produto da $1^{\text {a }}$ Conferência Internacional sobre Cuidados Primários à Saúde.

As décadas de 70 e 80 foram marcadas como um período conturbado do ponto de vista econômico, de reajuste social e político. A necessidade de expandir os mercados levou as nações a, aos poucos, abrirem seu comércio marcando o crescimento da ideologia econômica do liberalismo. Denominado de globalização, esse processo influenciou o comércio, as transações financeiras, os investimentos, a modernização tecnológica, a migração e movimento de pessoas e a disseminação de conhecimento ${ }^{12}$, além disso reemergiu outros desafios ambientais, como a mudança climática, a poluição do ar, exposição humana a situações insustentáveis.

Em 1986, a Carta de Ottawa como resultado da Conferência Internacional sobre Promoção da Saúde traz a promoção da saúde como fator fundamental de melhoria da qualidade de vida, com a inclusão e capacitação da comunidade nesse processo, não sendo essa responsabilidade exclusiva do setor saúde. Neste mesmo ano, a $8^{\text {a }}$. Conferência Nacional da Saúde (CNS) no Brasil considerou a saúde como resultante das condições de vida e do meio ambiente dos povos ${ }^{13}$. O movimento da reforma sanitária brasileira influenciou fortemente este processo visando novas práticas de saúde, embasadas na lógica dos pensamentos e movimentos mundiais para a promoção da saúde.

A $8^{a}$. CNS foi um dos principais momentos da luta pela universalização da saúde no Brasil. Um processo altamente participativo, democrático e representativo, contou com a participação de diferentes atores sociais. Na ocasião reuniram-se acadêmicos, profissionais da área de saúde, sindicatos de trabalhadores, movimentos populares de saúde e também pessoas não diretamente vinculados à saúde. O resultado foi a evidência da necessidade de modificações no setor saúde para além da reforma administrativa e financeira. Denominada reforma sanitária, obteve sua legitimação com a Constituição Federal de 1988, redefiniu o conceito de saúde como direito ${ }^{14}$. Esse marco representou uma ruptura inédita com a história anterior das políticas sociais brasileiras, ao garantir o acesso à saúde como direito social universal.

Em 1987 o Relatório denominado Nosso Futuro Comum dá destaque ao conceito de desenvolvimento sustentável que deveria envolver três dimensões: o cálculo econômico, os aspectos sócio-políticos e os aspectos biofísicos ${ }^{15}$. A Comissão Mundial sobre o Meio Ambiente e Desenvolvimento que publicou o relatório tinha a tarefa de definir uma agenda global para mudanças, com estratégias ambientais de longo prazos, por volta dos anos 2000. Porém, Tambellini (2013) destaca que elementos centrais que determinam as contradições diagnosticadas estão omissos no relatório, tais como a dominação político-econômico-militar assim como as explorações no interior de cada nação. 
Em 1990 a lei Orgânica do Sistema Único de Saúde (SUS) é aprovada. A saúde recebe a missão de garantir as condições de bem-estar físico, mental e social, e neste sentido mais tarde a Conferência das Nações Unidas sobre Meio Ambiente e Desenvolvimento (CNUMAD), denominada Rio-92, considerou um conjunto de ações de saúde e meio ambiente no contexto do desenvolvimento sustentável. Após a Conferência, e com a sensibilização mundial quanto à necessidade de desenvolvimento de políticas e estratégias sobre saúde e ambiente, foi realizada em 1995 a Conferência Pan-Americana sobre Saúde e Ambiente no Desenvolvimento Humano Sustentável (COPASAD). A COPASAD teve como resultado um documento contendo o Plano Regional de Ação no contexto do desenvolvimento sustentável orientador aos países que deveriam desenvolver seus planos específicos. Questões como a água e seus impactos à saúde, até este momento, eram tratadas do ponto de vista da engenharia de saneamento. A vigilância da água, e seus impactos à saúde, era timidamente realizada como demandas relacionadas à vigilância de doenças como diarreias e hepatites.

Na perspectiva de ampliar o olhar das ações em saúde, o Brasil então sistematizou seus principais problemas de saúde e ambiente apresentando diretrizes programáticas de referência para as ações de planejamento em saúde e meio ambiente no país em um documento denominado "Plano Nacional de Saúde e Ambiente no Desenvolvimento Sustentável - Diretrizes para Implementação", e em 2001 a VSA no Brasil começou a ser construída na estrutura formal do Ministério da Saúde (MS) do Brasil a partir deste documento nacional e propôs a execução de ações considerando o campo da Vigilância em Saúde e suas interfaces de atuação. Ainda na década de 90, o desenvolvimento de uma metodologia para a construção de indicadores de saúde ambiental como ferramenta para avaliação da determinação socioambiental da doença foi outro grande avanço, resultado do projeto Headlamp (Análise de Saúde e Ambiente para a tomada de decisões), a partir de uma colaboração entre o Programa das Nações Unidas para o Meio Ambiente (PNUMA), a Agência de Proteção Ambiental dos Estados Unidos da América e a Organização Mundial da Saúde (OMS) ${ }^{16,17}$.

No espaço político, atualmente predominam os interesses das grandes corporações nacionais, do sistema financeiro, de latifundiários, de empresas multinacionais e por esta razão permanece o desafio da construção de uma perspectiva ecológica, solidária e democrática. As políticas de indução e de acolhimento às demandas econômicas e sociais não dependem necessariamente das ações desenvolvidas pela vigilância em saúde. No cenário prevalece uma governança voltada ao desenvolvimento de forças produtivas com forte acúmulo de poder político dos empreendedores e promotores da política econômica associadas a uma precária política ambiental de sustentabilidade vinculada a uma política social redistributiva de cunho assistencial no campo da saúde e da assistência social.

Nesse contexto há uma pressão pela desregulação ambiental e social associada ao uso de tecnologias do agronegócio, para geração de energia e polos empresariais, com forte impacto 
negativo no ambiente e consequentemente na saúde. Ainda, este cenário impacta visivelmente o espaço do trabalho tendo implicações diretas na saúde do trabalhador.

Por ser dinâmico e complexo o atual cenário exige análise contínua da situação da saúde das populações a fim de orientar a tomada de decisão, auxiliando na redefinição de prioridades, predição de cenários futuros, estruturação do setor saúde e avaliação das intervenções implementadas.

\section{Aspectos teóricos para a VSA no Brasil}

Para o olhar do ambiente, Tambellini ${ }^{18}$ propõem três conceitos que orientam a relação com a saúde: (i) o ambiente como exterioridade ao homem; (ii) : o ambiente como sistema ecológico; e (iii) o ambiente como sistema socioecológico. No primeiro modelo o ambiente tido como natural, é explicado do ponto de vista estritamente biológico e é percebido como condições físicas, químicas, biológicas e sociais individualizadas, e estabelece com o homem uma relação agressor-agredido.

No segundo o ambiente é entendido como um sistema ecológico, complexo e composto por elementos bióticos e abióticos, organizado hierarquicamente (determinado pelo fluxo alimentar). Este modelo permite um entendimento de relação ambiente-saúde.

O terceiro considera o ambiente como sistema socioecológico. Este último, mantém a organização hierárquica do modelo anterior e incorpora os componentes sociais das coletividades humanas. Neste modelo, o homem é considerado um animal sociopolítico que transforma, a seu favor, a natureza e os sistemas ecológicos, utilizando técnicas de produção para o consumo, desnaturalizando o meio exigindo a reelaboração do conceito de ambiente, conforme tradicionalmente elaborado pela ecologia. É exatamente esta intervenção que transforma o ecossistema em um sistema socioecológico.

Foi no campo da Vigilância em Saúde que as ações relacionadas aos impactos do meio ambiente na saúde tomaram forma. Este campo é utilizado para vigiar pessoas no tempo e lugar, controlar contextos e fazer a promoção da saúde a partir dos efeitos ou situações de doença, dos perigos e das formas de exposição, individuais ou coletivas ${ }^{19}$, e é considerada uma das funções essenciais da saúde pública contemporânea ${ }^{20}$.

Em revisão de bibliografia recente realizada por Netto et $\mathrm{al}^{21}$, o termo vigilância vem do idioma inglês, surveillance, que tem origem no idioma francês, sur (sobre) e veiller (observar) ${ }^{22}$. Para $\mathrm{Choi}^{23}$ a vigilância em saúde pode ser representada, em sua ampla revisão como do passado, do presente e do futuro em vigilância em saúde pública.

O passado da vigilância em saúde se consistiu no registro das grandes epidemias da história da humanidade e data da época do Faraó Memphis, quando a primeira epidemia foi registrada na história da humanidade, ocorrida em 3.180 a.C. De acordo com os registros, três tipos de informação foram incluídos nos registros históricos das epidemias: desfechos de saúde, fatores de risco e intervenções. Informações estas ainda essenciais para a saúde pública atual. Já nesta 
época eram realizadas coletas e análises de dados e a partir da ideia de Hipócrates que acreditava que as doenças eram causadas pela natureza de um determinado lugar. A coleta de dados deveria considerar o lugar o território, o ambiente natural e as pessoas para determinar as doenças ${ }^{21}$.

O termo vigilância foi utilizado inicialmente em saúde pública para descrever o monitoramento próximo de pessoas que, devido a uma exposição, estavam sob o risco de desenvolver doenças altamente contagiosas e infecciosas. Estas pessoas eram monitoradas de forma que, caso exibissem sintomas da doença seriam postos em quarentena para prevenir a disseminação da doença.

Em 1963 o Center of desease Control (CDC), publicou artigo ${ }^{24}$ no qual definia a vigilância de uma doença como de necessidade contínua, com a observação sobre a distribuição e as tendências da incidência, coletas sistemáticas, consolidação e avaliação de relatórios de morbidade e mortalidade e outros dados relevantes. As definições mais recentes de vigilância, incluindo a de 2001 do CDC, a de 2006 da Agência de Saúde Pública do Canadá, bem como a de 2013 do Brasil enfatizam a vigilância em saúde como "ação em saúde pública".

No Brasil, ações de vigilância, prevenção e controle das doenças foram organizadas durante o século XX como programas verticalizados, com a formulação, a coordenação e a execução de ações realizadas diretamente pelo Governo Federal. Esses programas estabeleceram-se como serviços nacionais para o controle de doenças mais prevalentes da época. Sua estrutura dava-se sob forma de campanhas, adaptando-se a uma época em que a população era majoritariamente rural e os serviços de saúde escassos e concentrados, quase que exclusivamente, nas áreas urbanas. Atualmente, a partir de 2003, com a reorganização do MS a área que era destinada às ações de epidemiologia e controle de doenças reúne todas as ações de vigilância, prevenção e controle de doenças numa mesma estrutura, consolidando o processo de ampliação do objeto da vigilância. O objetivo foi buscar responder melhor aos desafios apresentados pelo complexo perfil epidemiológico do Brasil contemporâneo ${ }^{25}$.

De acordo com Silva Jr. ${ }^{25}$ a adoção do conceito de vigilância em saúde no Brasil procura simbolizar uma nova abordagem, mais ampla do que a tradicional prática de vigilância epidemiológica, e inclui: a) a vigilância das doenças transmissíveis; b) a vigilância das doenças e agravos não transmissíveis e seus fatores de risco; c) a vigilância ambiental em saúde; e d) a vigilância da situação de saúde, correspondendo a uma das aplicações da área também denominada como análise de situação de saúde.

\section{Desafios da VSA no Brasil - Uma epidemiologia situada}

As questões relacionadas ao meio ambiente e sua influência na saúde estão em evidência junto aos movimentos sociais e à academia, assim como na construção e implementação de uma política pública que dê conta dos aspectos abordados na relação do ambiente com a saúde.

A compreensão das relações entre desenvolvimento, ambiente e saúde, seja pela ciência, pelo poder público ou pela cidadania, com o sentido de identificar seus processos críticos é um 
desafio de forma a possibilitar a elaboração e produção de instrumentos capazes de articular ações relacionadas à natureza dessa relação, planejar ações estratégicas e mecanismos para a promoção da saúde. A abordagem deverá considerar aspectos de natureza conceitual, científica e técnica, assim como aqueles inerentes às políticas e intervenções que se relacionem às articulações entre 0

modelo de desenvolvimento, os sistemas socioecológicos e a saúde das coletividades ${ }^{26}$.

Temos que a Saúde Pública no Brasil, atualmente, traz em sua história a forma de se organizar e agir a partir de teorias que enfatizam as dimensões biológicas e individuais ao analisar tendências relativas à distribuição de riscos, doenças, incapacidades e mortes nas populações, e também teorias com ênfase nas relações sociais e ecológicas, resultando em estratégias de planejamento e políticas públicas que enfatizam desde a eficiência de tecnologias, serviços de saúde assistenciais e medidas pontuais de prevenção, até a promoção a saúde como resultado do modo de produção e consumo.

A ciência normal ${ }^{27}$, com sua perspectiva biomédica e epidemiológica clássica produziu e reproduz, uma forma de pensar e agir que restringe a dimensão social na determinação do processo saúde-doença, levando ao entendimento da sociedade como um agregado de indivíduos com características quantificáveis, sem priorizar e articular melhorias das condições de vida, do trabalho e suas estruturas sociais ${ }^{28}$. Pensar e agir a partir de relações entre grupos ao invés de isolar efeitos sobre indivíduos tem sido o desafio enfrentado no campo da Saúde Coletiva, em especial a partir do movimento sanitário brasileiro com influência latino-americana do campo da medicina social, requer mudança de perspectiva e principalmente de referencial teórico, com um olhar para as desigualdades sociais e de saúde, o que demanda maior precisão conceitual nos estudos de condições de vida e situações de saúde.

Compreender as relações entre os determinantes sociais e a saúde é fundamental para caminhar na perspectiva de se trabalhar e incorporar os conceitos de diferença, distinção, desigualdades, diversidade, iniquidade e equidade, vulnerabilidade e exposição. As abordagens teóricas e práticas podem ser diferentes a partir de diferentes perspectivas, ainda que alguns autores reduzam as desigualdades em saúde, dentre outros aspectos da determinação da saúde, a dimensões puramente individuais, pois consideram a abordagem social pouco científica ${ }^{29}$.

Discutir fatores gerais de natureza social, econômica, política e suas mediações, permite identificar onde e como devem ser feitas as intervenções com maior impacto, sendo necessário o fortalecimento conceitual e metodológico de abordagem dos determinantes da saúde nos indivíduos e nas populações. Os fatores individuais são importantes para identificar que indivíduos no interior de um grupo estão submetidos a maior risco. E há os enfoques que buscam analisar as relações entre a saúde das populações, as desigualdades nas condições de vida e o grau de desenvolvimento da trama de vínculos e associações entre indivíduos e grupos ${ }^{30}$.

Questões como a vulnerabilidade às doenças, a exposição ambiental e seus efeitos sobre a saúde distribuem-se de maneira diferente segundo os indivíduos, regiões e grupos sociais e 
relacionam-se com a pobreza, com as crises econômicas e com o nível educacional, por exemplo. Produzir conhecimento neste campo envolve a investigação sob as trajetórias sociais, interação e os contextos, incorporando os fatores econômicos, políticos e culturais, numa dada sociedade, assim como as várias maneiras de atuação e de relação entre os indivíduos ${ }^{31}$.

Nas últimas três décadas, houve o reconhecimento da crise socioambiental e dos riscos ecológicos globais, gerando noções como desenvolvimento sustentável e acordos internacionais que pautam agendas políticas, movimentos sociais, políticas públicas e a própria saúde coletiva $^{32}$. Neste sentido, o movimento de enfrentamento dos paradigmas e racionalidades hegemônicos da biomedicina, da biotecnologia e da epidemiologia clássica, bem como o resgate e a atualização do debate teórico e político que fundou a Medicina Social Latino Americana e a Saúde Coletiva ${ }^{33}$, tem pautado as instituições de produção do conhecimento no sentido de avançar na compreensão da situação de saúde, seus determinantes, contextos e identificação, construção e consolidação de políticas públicas e objetos de pesquisas como alternativas de produção de conhecimentos que incorporem as populações e movimentos sociais como sujeitos coletivos, com seus saberes e projetos de sociedade, de forma intra e intersetorial.

Na última década, a mudança na estrutura econômica brasileira e a elaboração de grandes projetos de crescimento para o seu desenvolvimento, evidenciaram a necessidade de atuação do setor saúde de forma intensificada, em função das transformações na vida das pessoas. O amplo contexto da desigualdade na sociedade torna o processo por vezes isolado e interminável. Os elementos a serem considerados na relação entre o desenvolvimento econômico, social e ambiental estão além da visão biologicista da ciência normal. O caminho metodológico aponta para a criação de modelos teóricos que permitam a leitura dos territórios e dos processos de trabalho de forma interdisciplinar entendendo a complexidade dos objetos, dialogando com os saberes científicos e populares, maximizando os ganhos em saúde.

De acordo com Kuhn $(2011)^{34}$, um paradigma indica que precisa ser ajustado quando surgem acontecimentos que a ciência não pode, com seus atuais instrumentos, compreender. Esse cenário de crise planetária, com um conjunto de crenças, valores e métodos científicos reducionistas que não abrangem a complexidade de seus objetos e fenômenos, demanda um novo olhar cientifico.

Alguns epistemólogos contemporâneos qualificam esta nova situação como um desafio da complexidade $^{35}$, ou seja, como uma situação em que existe a necessidade de se pensar e agir não em termos de objetos, mas em termos de relações. Assim, o complexo seria a marca de "aquilo que está junto", como é o caso dos organismos vivos, dos grupos, das populações e do ambiente ${ }^{36}$.

O método de saber distinguir sem separar e juntar sem confundir ${ }^{37}$, ou seja, de saber fazer, por um lado, as distinções necessárias entre âmbitos de pertinência diferentes e, por outro lado, de detectar os vínculos entre eles, são necessários e significativos para dar conta das práticas simbólicas humanas ${ }^{38}$. 
Fortalecer a discussão do modelo teórico da relação Saúde, Ambiente e Sustentabilidade, instrumentalizar sua operacionalização, via vigilância em saúde ambiental, foi um desafio assumido pelo Brasil durante a última década, particularmente pela Fundação Oswaldo Cruz. Dentre os desafios, ainda hoje em processo de superação, estão a necessidade de formação de equipes multidisciplinares, além da construção de sistemas de informação capazes de auxiliar a análise de situações de saúde e a tomada de decisões, o desenvolvimento de tecnologias sociais a serem incorporadas pelo SUS, as dimensões da produção de conhecimento (discussão teórica), da política, da governança e do controle social, devendo estar articuladas ao processo operacional, com ênfase na vigilância em saúde.

No espaço político predominam os interesses das grandes corporações nacionais, do sistema financeiro, de latifundiários, de empresas multinacionais, e por esta razão permanece o desafio da construção de uma perspectiva ecológica, solidária e democrática. As políticas de indução e de acolhimento às demandas econômicas e sociais não dependem necessariamente das ações desenvolvidas pela vigilância em saúde. Esta é parte do processo e incorpora elementos para as decisões, influencia e é condicionada pela disputa política.

No cenário atual prevalece uma governança voltada ao desenvolvimento de forças produtivas com forte acúmulo de poder político dos empreendedores e promotores da política econômica associadas a uma precária política ambiental de sustentabilidade vinculada a uma política social redistributiva de cunho assistencial no campo da saúde e da assistência social. Há uma pressão pela desregulação ambiental e social associada ao uso de tecnologias do agronegócio, para geração de energia e polos empresariais, de forte impacto negativo no ambiente e consequentemente na saúde. Ainda, este cenário impacta visivelmente o espaço do trabalho tendo implicações diretas na saúde do trabalhador.

Por ser dinâmico e complexo esse cenário exige análise contínua da situação da saúde das populações de forma a orientar e subsidiar a tomada de decisão, auxiliando na redefinição de prioridades, predição de cenários futuros, estruturação do setor saúde e avaliação das intervenções implementadas de forma rotineira.

\section{REFERÊNCIAS BIBLIOGRÁFICAS}

1 ROSEN, G. Uma História da Saúde Pública. São Paulo, Rio de Janeiro: Hucitec, Unesp, Abrasco, 1994

2 BATISTELlA, C. Saúde, doença e cuidado: complexidade teórica e necessidade histórica. In: FONSECA, Angélica Ferreira; CORBO, Anamaria D’Andrea. (Orgs.). O território e o processo saúde doença. Rio de Janeiro; EPSJV, 2007. 265: 25-49.

3 FOUCAULT, M. O nascimento da medicina social. In: FOUCAULT, M. Microfísica do Poder. 3.ed. Rio de Janeiro: Graal, 1982b 
4 GUTIERREZ, P. R. \& OBERDIEK, H. I. Concepções sobre a saúde e a doença. In: ANDRADE, S. M. de; SOARES, D. A. \& CORDONI JUNIOR, L. (Orgs.) Bases da Saúde Coletiva. Londrina: UEL, 2001.

5 TAMBELlini, A; MIRANDA, A. Saude e Ambiente - In:Políticas e Sistema de Saúde no Brasil. Giovanella L, Escorel S, Lobato LVC, Noronha JC, Carvalho AI, organizadores. $2^{\mathrm{a}}$ Ed. Rio de Janeiro: Editora Fiocruz; 2013: 1037-1073.

6 CZERESNIA, D. Do Contágio à Transmissão: ciência e cultura na gênese do conhecimento epidemiológico. Rio de Janeiro: Fiocruz, 1997.

7 PALMEIRA, G. et al. Processo saúde doença e a produção social da saúde. In: EPSJV. (Org.) Informação e Diagnóstico de Situação. Rio de Janeiro: Fiocruz, EPSJV, 2004. (Série Mate-rial Didático do Programa de Formação de Agentes Locais de Vigilância em Saúde Proformar)

8 RIOS, J. L. P. - Poluição e degradação ambiental - LNEC - Lisboa, 1974

9 Lalonde M. A new perspective on the health of Canadians. A working document. Ottawa: Government of Canada, 1974

10 Minkler M. Health education, health promotion and the open society: an historical perspec-tive. Health Educ Q 1989 Spring;16(1):17-30

11 Frohlich KL, Potvin L. Transcending the known in public health practice. The inequality paradox: the population approach and vulnerable populations. Am J Public Health 2008 Feb;98(2):216-21

12 International Monetary Fund. (2000). "Globalization: Threats or Opportunity." 12th April 2000: IMF Publications.

13 Brasil. Ministério da Saúde. Conselho Nacional de Saúde. Subsídios para a construção da Política Nacional de Saúde Ambiental / Ministério da Saúde, Conselho Nacional de Saúde. Bra-sília: Editora do Ministério da Saúde, 2007

14 Brasil. VII Conferencia Nacional de Saúde. Relatório Final. Disponível em http://conselho. saude.gov.br/biblioteca/relatorios/relatorio_8.pdf. Acesso em 24. fev.2015

15 Moreira, R. J. Críticas ambientalistas a revolução verde. Estudos Sociedade e Agricultura.

15:39-52, out 2000 . 
16 Corvalán, C.; Briggs, D.; Kjellstrom, T. Development of environmental health indicators. In: Briggs, D.; Corvalan, C.; Nurminen, M. Linkage methods for environment and health analysis. General guidelines. Genebra: UNEP, USEPA, WHO, 1996

17 Domingues, E. Indicadores de sustentabilidade para gestão dos recursos hídricos no Brasil. Relatório de consultoria ao Centro Internacional de Desenvolvimento Sustentável, Fundação Getú-lio Vargas e Escola Brasileira de Administração Pública. Rio de Janeiro: FGV; CIDS; EBAP, 2000

18 Tambellini, A; Miranda, A. Saúde e Ambiente - In: Políticas e Sistema de Saúde no Brasil. Giovanella L, Escorel S, Lobato LVC, Noronha JC, Carvalho AI, organizadores. 2ªd. Rio de Ja-neiro: Editora Fiocruz; 2013: 1037-1073.

19 Paim JS. Epidemiologia e planejamento: a recomposição das práticas epidemiológicas na gestão do SUS. Ciência \& Saúde Coletiva 8(2):557-567. 2003

20 WHO/PAHO. Essential Public Health Functions. http://www.paho.org/hq/index.php?option=com_content $\&$ view=category \&layout=blog\&id=3175\&Itemid=3617 Acesso em 13.jan.2014.

21 Netto, Guilherme Franco; VillardI, Juliana; et ali. Termo de Referência - Vigilância em Saúde - Tópicos para serem abordados, Reunião de 14/10/2013. (Mimeo).

22 BrachmaN, P. S. Public health surveillance. In: Brachman, P. S.; Abrutyn, E. (Eds.). Bacte-rial infection of humans: epidemiology and control. New York: Springer, 2009.

23 Choi, B. C. K. The past, present and future of public health surveillance. Scientifica, v. 2012, 2012: 26.

24 Langmuir, A. D. The surveillance of communicable diseases of national importance. N. Engl. J. Med., v. 268, p. 182-92, 1963

25 Silva Júnior, J B. Epidemiologia em serviço: uma avaliação de desempenho do Sistema Nacional de Vigilância em Saúde. 318 f. Tese (Doutorado em Saúde Coletiva) - Faculdade de Ci-ências Médicas, Universidade Estadual de Campinas. Campinas, SP, 2004.

26 Tambellini, A; Miranda, A. Saúde e Ambiente - In: Políticas e Sistema de Saúde no Bra-sil. Giovanella L, Escorel S, Lobato LVC, Noronha JC, Carvalho AI, organizadores. $2^{\mathrm{a}}$ Ed. Rio de Janeiro: Editora Fiocruz; 2013: 1037-1073

27 Kuhn, T. S. A estrutura das revoluções científicas. São Paulo: Editora Perspectiva, 1987.

28 Porto, M F Sa; Rocha, D F; Finamore, R. Saúde coletiva, território e conflitos ambientais: bases para um enfoque socioambiental crítico. Ciênc. Saúde coletiva, 2014, 19 (10): 4071-4080.

29 Barata, R. Iniquidade e saúde: a determinação social do processo saúde-doença Revista USP, São Paulo, 2001, 51:138-145. 
30 Buss, Paulo, M. \& Pelegrini Filho, Alberto. - A saúde e seus determinantes sociais. Phy-sis: Revista Saúde Coletiva, Rio de Janeiro, 17(1):77-93, 2007.

31 Muñoz Sanchez, A.I. e Bertolozzi, M. R. - Pode o conceito de vulnerabilidade apoiar a construção do conhecimento em saúde coletiva?. Revista Ciência e Saúde Coletiva, 12(2):319324. 2007

32 Porto, MFS; Rocha, DF; Finamore, R. Saúde coletiva, território e conflitos ambientais: bases para um enfoque socioambiental crítico. Ciênc. Saúde coletiva 2014, 19 (10): 4071-4080.

33 Carta Política do $2^{\circ}$ Simpósio Brasileiro de Saúde e Ambiente. Belo Horizonte, 22 de outubro de 2014. Acesso em 02.dez.2014. Disponível em http://www.sibsa.com.br/informativo/ view?TIPO=\&ID_INFORMATIVO=103

34 Kuhn, T. S. A estrutura das revoluções científicas. 11.ed. São Paulo: Perspectiva, 2011. 264 p.

35 Bocchi, G. \& Ceruti, R., 1987. La sfida della Complessità. Milano: Feltrinelli.

36 Schramm, F R.; Castiel, L D. Processo saúde/doença e complexidade em epidemiolo-gia. Cad. Saúde Pública, Rio de Janeiro, 1992, 8 (4).

37 Morin, E. Introduction à la Pensée Complexe. Paris: ESF, 1990.

38 Schramm, F R. Interpretação complexa da lei de hume e da falácia naturalista. Mimeo. 2011. 